\title{
The effect of Steri-Strip orientation on wound healing: A survey of current applications and trends amongst
}

\section{surgeons}

Yaeesh Sardiwalla', Khader Zimmo', Jason Williams, MD², Michael Bezuhly, MSc, $\mathrm{MD}^{2}$

I. Class of 2019, Faculty of Medicine, Dalhousie University, Halifax, Nova Scotia, Canada.

2. Division of Plastic and Reconstructive Surgery, Dalhousie University, Halifax, Nova Scotia, Canada.

\section{Abstract}

Wound closure strips are used in the repair of superficial, low tension wounds, lacerations, and surgical incisions. They represent an inexpensive, easy, and painless way to close such wounds or as an adjunct in a multi-layered closure. There is debate over correct orientation (transverse, oblique, zig-zag or parallel) and importance of wound closure strips relative to the incision. The purpose of this research was to examine the closure techniques amongst Canadian plastic surgeons and current applications of wound closure strips using an online survey circulated to members of the Canadian Society of Plastic Surgery. Participants answered a series of multiple choice and short answer questions regarding their practice, closure techniques and use of wound closure strips. A total of I 20 plastic surgeons (30.0\%) completed the survey with $\mathbf{8 9 . 9 \%}$ reporting wound closure strips usage. Of respondents who used wound closure strips, $57.2 \%$ used them in more than half of all wound closures. Parallel orientation to the wound (52.3\%) and perpendicular orientation (30.8\%) were the most common techniques employed, and these were applied in a consistent fashion $(90.6 \%)$ covering the whole wound (78.1\%). Most plastic surgeons agreed there is a need to further study adhesive strips. This survey of Canadian plastic surgeons has demonstrated the variability that exists in wound closure strip application technique, orientation and intended function. These findings suggest further investigation of wound closure strip usage may be needed to help guide clinical practice.

$\mathrm{T}_{\mathrm{t}}^{\mathrm{t}}$ he four most commonly used wound closure techniques are adhesive strips, sutures, staples, and surgical glue, with the former two options being the most commonly employed. ${ }^{1}$ Wound closure strips are mostly used to provide additional wound support after suture removal or as an adjunct with buried dermal or absorbable running subcuticular sutures in low-tension wounds to add stability to the site. ${ }^{2}$ Studies have assessed the use of wound closure strips for the repair of surgical skin incisions and traumatic lacerations, assessing the relative effectiveness compared to other common modalities. ${ }^{3-5}$ When comparing adhesive strips used with buried sutures to traditional layered closures, no difference was seen in scar contour, erythema and overall cosmesis between the two methods for surgical incisions. ${ }^{3}$ In the short term, wound closure strips can show improved cosmetic outcomes and patient satisfaction for certain wounds; however, both methods provide the same long-term cosmetic results. ${ }^{4,5}$ Wound closure strips are therefore sometimes considered a time- and cost-effective alternative to sutures for both traumatic lacerations and surgical incisions.

Wound closure strips, such as Steri-Strips ${ }^{\mathrm{TM}}\left(3 \mathrm{M}^{\circledR}\right.$, Two Harbours, MN, USA), are a form of adhesive tape used in the closure of superficial, low tension wounds, lacerations, and surgical incisions. ${ }^{1}$ Given their ease of application, wound closure strips are used in a variety of settings, including the emergency department and operating suite. Wound closure strips are inexpensive, easy, and painless to apply, which is why they are sometimes preferred to other forms of wound closure methods such as sutures or staples in conscious patients. When compared to cutaneous sutures, wound closure strips provide faster closure times, avoid visible puncture marks, and decrease the risk of tissue strangulation and needle-stick injuries. ${ }^{3}$ Additionally, follow-up visits for suture removal are not required when using wound closure strips, making them a favourable and cost-effective option.

There have been several observational studies and opinions published on the topic of optimal application for adhesive tapes. ${ }^{6-8}$ The authors are unaware of any clear consensus or randomized control trial reported supporting the use of one orientation (i.e. transverse, oblique, zig-zag or parallel) for wound closure strips over another relative to the incision. A study in healthy volunteers assessed Steri-Strips ${ }^{\mathrm{TM}}$ adherence over time for different patterns of parallel orientations (no edges, tacked, overlapping). The study found that the application of strips in a parallel, non-overlapping fashion after complete coating of the skin surface with mastisol had the best adherence over time on healthy skin. ${ }^{7}$ It has also been reported that transverse application of adhesive strips in high tension areas such as the back after a latissimus dorsi flap were more susceptible to shearing effects and subsequent 
Table I: The complete list of questions and answer choices included in the distributed survey

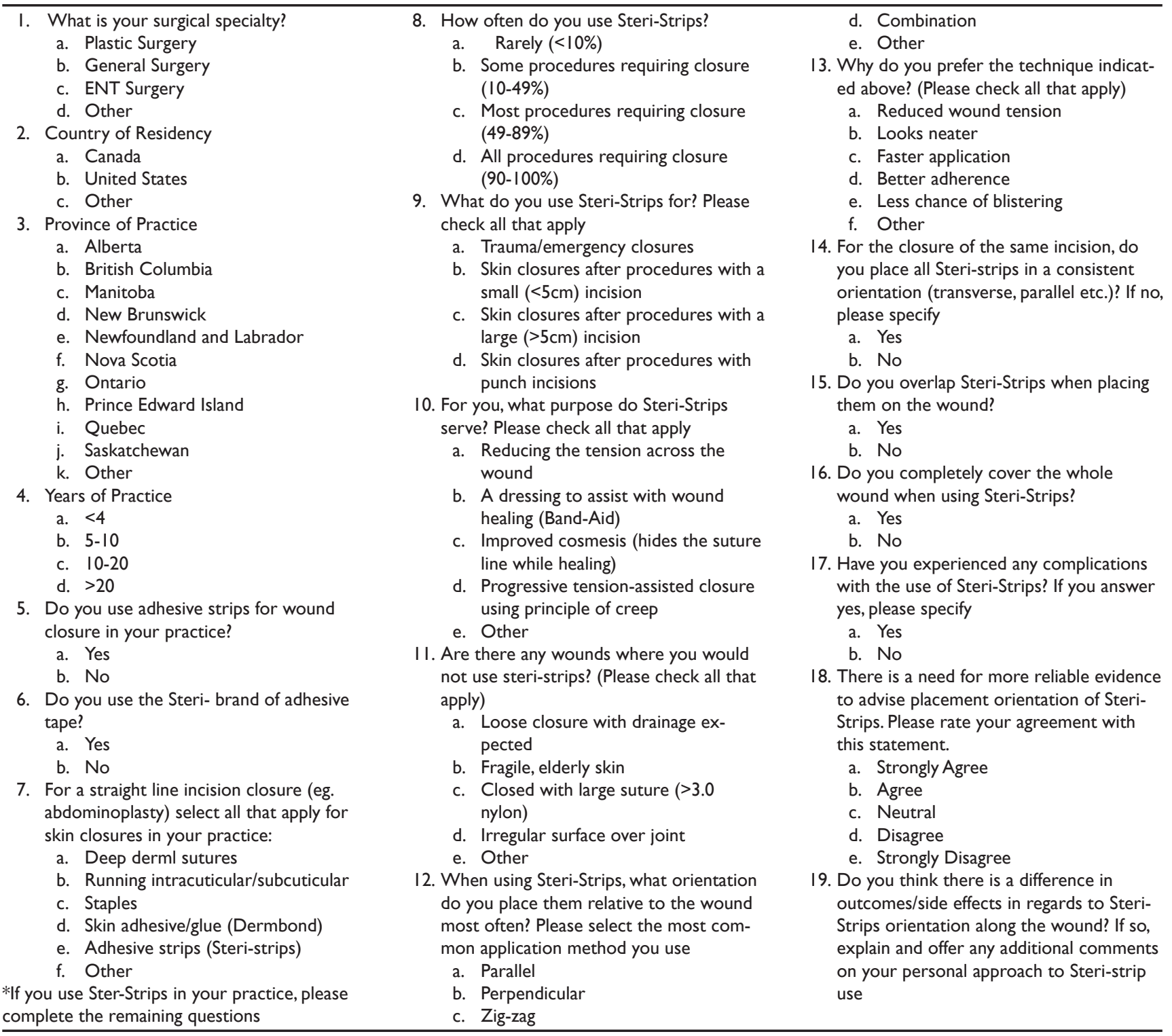

blistering. ${ }^{8}$ For these anecdotal reasons, it is believed that application of adhesive tapes parallel to wounds maintains good adherence and avoids blistering complications. $^{7,8}$

While the manufacturer of Steri-Strips ${ }^{\mathrm{TM}}$ advises that they be applied transversely across the wound with supporting (tacked) parallel strips applied away from the incision forming a railroad pattern, there is variability in clinical practice since the rationale and outcome evidence for this method is lacking. ${ }^{8}$ The purpose of this study was to examine variability in closure techniques among Canadian plastic surgeons and current applications trends of wound closure strips.

\section{Methods}

Institutional ethics approval was obtained for this study. The study was unfunded. A total of 429 plastic surgeons identified as active members of the Canadian Society of Plastic Surgeons (CSPS) were invited to participate in this study. An introductory email was sent to CSPS members via the Society's web server list. This included the informed consent form and a link to participate in the study. All data captured was anonymous and only surveys from a unique IP address were included in the subsequent analysis to avoid multiple responses by the same participant. Participants answered a series of multiple choice and short answer questions regarding their practice, closure techniques and use of wound closure strips (Table 1). More specifically, the survey evaluated frequency of wound closure strip use, orientation of placement, perception of function and complications of use. The responses were saved and analyzed on a secure Dalhousie server using Opinio ${ }^{\mathrm{TM}}$ (ObjectPlanet ${ }^{\oplus}$, Oslo, Norway). 
Survey answers were calculated as a relative frequency percentage to identify trends in wound closure strip use. For each question, the total number of participants that selected an answer choice relative to the total number of participants that answered that question was calculated and expressed as a percentage. Any individual survey response with incomplete information was not included in the analysis.

\section{Results}

A total of 120 plastic surgeons (30.0\%) completed the survey according to the inclusion criteria. The majority (96.7\%) were Canadian surgeons from Ontario (38.3\%, Figure 1). The majority of respondents $(74.2 \%)$ had been in practice for greater than 10 years (Table 2). Wound closure strip use was used by $89.9 \%$ of respondents, with $82.9 \%$ using the Steri-Strip ${ }^{\mathrm{TM}}$ brand specifically.

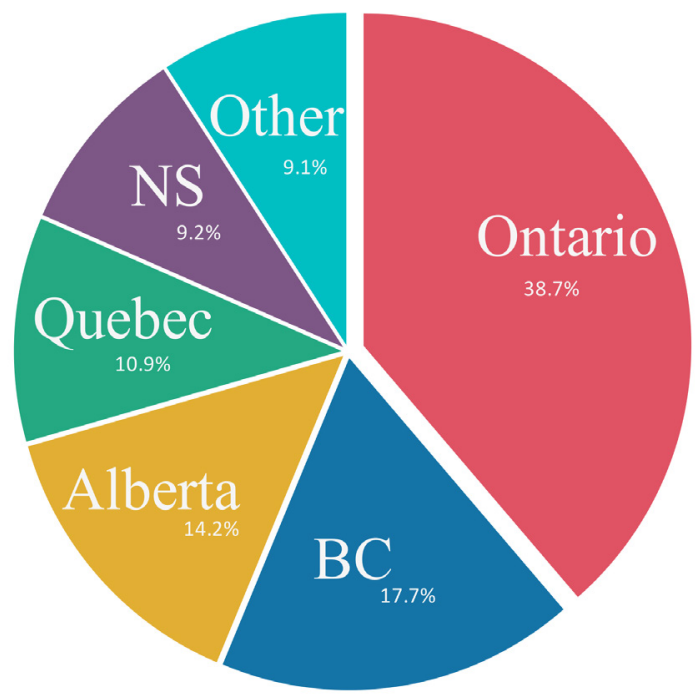

Figure I: The geographic location of survey respondents practice

The most common methods employed for closure of a straight-line incision such as abdominoplasty were deep dermal sutures (94.2\%) and running subcuticular/ intracuticular $(94.2 \%)$ followed by the application of wound closure strips. Use of skin glue (22.3\%), micropore tape $(7.5 \%)$ and staples $(2.5 \%)$ were also reported (Table 3).

Table 2: The geographic location of survey respondents practice

\begin{tabular}{lll}
\hline & Answer Chosen & $\begin{array}{l}\text { Positive Response } \\
\text { Frequency }\end{array}$ \\
\hline \multirow{2}{*}{ Training } & Plastic Surgery & 100 \\
& Canadian Residency & 98.31 \\
\hline \multirow{4}{*}{ Experience } & $<5$ years & 14.29 \\
& $5-10$ years & 11.76 \\
& $10-20$ years & 29.41 \\
& $>20$ years & 44.54 \\
\hline
\end{tabular}

Table 3: Frequency of methods employed for the closure of a straight-line incision

\begin{tabular}{lll}
\hline & Answer Chosen & $\begin{array}{l}\text { Positive Response } \\
\text { Frequency }\end{array}$ \\
\hline & Deep Dermal & 94.17 \\
& Running subcuticular & 94.17 \\
Closure & Use of adhesive strips & 89.92 \\
Method & Steri-strips & 82.91 \\
& Skin Glue & 21.67 \\
& Staples & 2.5 \\
\hline
\end{tabular}

In the group that used wound closure strips, $57.2 \%$ used them in more than half of all wound closures. These included large incisions (88.6\%), small incisions (86.7\%), traumatic lacerations $(22.9 \%)$ and punch biopsy incisions (13.3\%). The strips were thought to function as a dressing (66.4\%), reduce tension (64.5\%) and improve cosmesis by hiding sutures (54.2\%). With regard to the orientation in which wound closure strips were applied relative to the incision, parallel orientation (52.3\%) and perpendicular orientation (30.8\%) were the most common techniques employed, and these were applied in a consistent fashion (90.6\%) covering the whole wound (78.1\%). Some surgeons (56.2\%) described overlapping the strips when applying them (Figure 2). Seventy percent of surgeons experienced complications with wound closure strips, most commonly blistering (82.7\%). Most plastic surgeons (73.9\%) were neutral, agreed or strongly agreed that more reliable evidence was required to determine if wound healing outcomes differed depending on how wound closure strips were applied relative to the incision.

\section{Discussion}

This survey of Canadian plastic surgeons has demonstrated that deep-dermal and running subcuticular/intracuticular sutures are most commonly used for wound closure. It is evident that adhesive strips such as Steri-Strips ${ }^{\mathrm{TM}}$ are often used as an adjunct to these methods forming part of the multi-layered closure utilized in both traumatic and elective surgical incisions. There was no definite variation in these trends according to geographic location, years of experience or country of training for the plastic surgeon.

The results of this study demonstrate substantial disagreement on how wound closure strips are applied. While the manufacturer of Steri-Strips ${ }^{\mathrm{TM}}$ recommends that they be applied in a non-overlapping and transverse orientation relative to the incision line with supporting strips away from and parallel to the incision line, no justification for this recommendation is provided. ${ }^{9}$ Although most surgeons (52.3\%) apply wound closure strips parallel to the wound, a large number (30.8\%) still apply them perpendicular. Therefore, there is poor adherence to the manufacturer's recommendations. The variability in practice is likely based on anecdotal experience. Based on the authors limited literature 

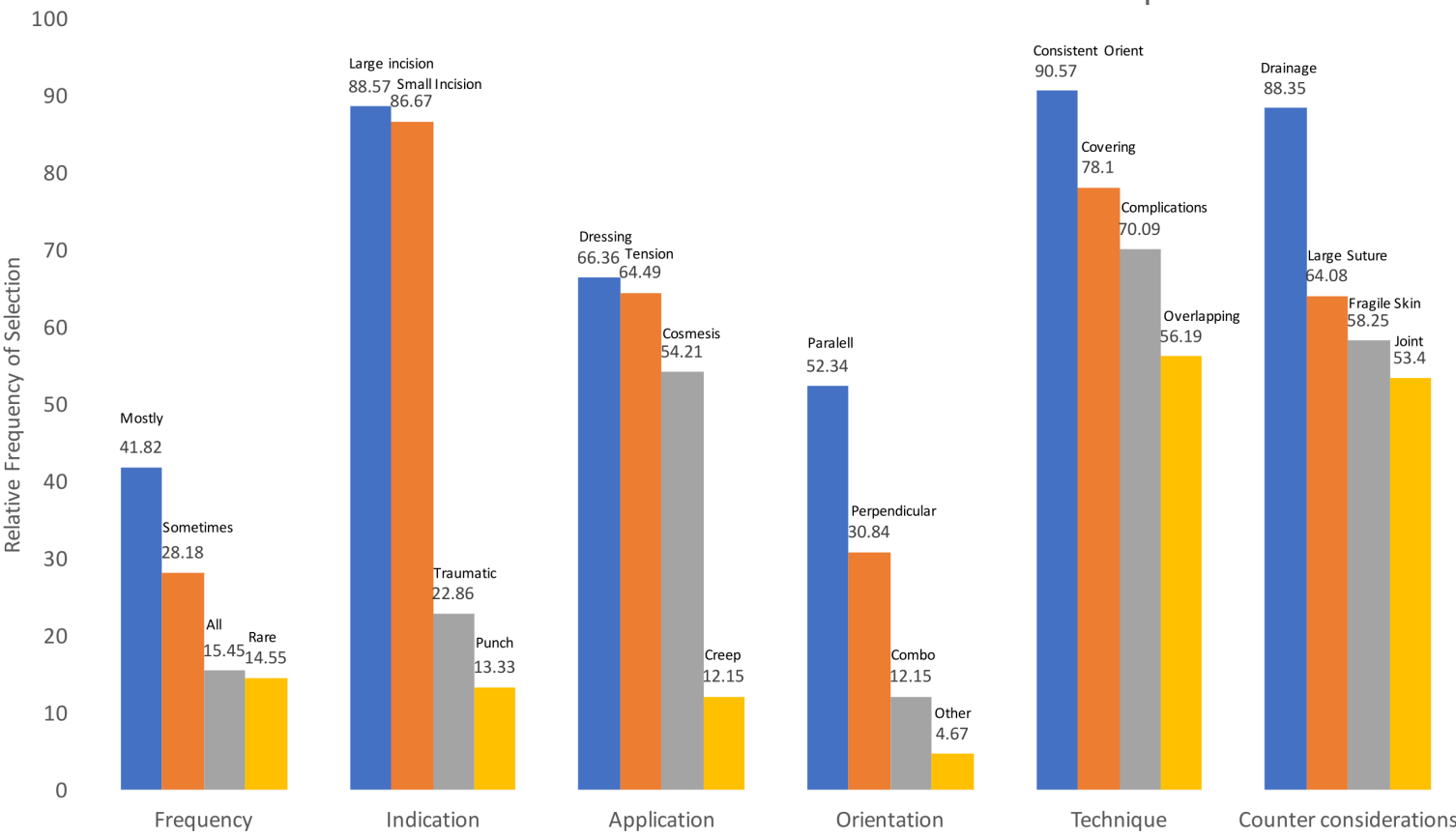

Figure 2: A description of how adhesive tapes are used by plastic surgeons that utilize them as part of wound closure

search, they are unaware of any study demonstrating superiority between one method of application compared to another.

The importance of these findings becomes evident when considering how wound closure strip application technique may affect wound healing. Scar appearance may be different based on the method selected. Furthermore, using wound closure strips is not an entirely benign component of wound closure. Orientation could be a determinant in complications such as blistering, where perpendicular placement is thought to play a larger role. As with any surgical intervention, an evaluation of risks and benefits must be weighed to justify usage in a scenario. Many surgeons responding to the survey agreed that there is a need to study wound closure strip use to determine what benefit, if any, it provides.

The study's limitations included a low response rate $(30.0 \%)$ and potential selection bias that must be interpreted accordingly. The survey's self-reported design has an associated reporting bias that fits a plastic surgeon's perspective of the event rather than an objective observational account. The wounds encountered by plastic surgeons may differ from those in the emergency room or general practitioner's office, but the principles of multi-layered technique still apply to all wounds that can be closed primarily. Plastic surgeons are often consulted in complex wound situations and difficult closures, so we believe the methods employed commonly in the survey are applicable to most wounds (deep and superficial) requiring a skin closure. The survey's results prompt the need for more reliable evidence with regard to correct adhesive strip application orientation. Although a formal literature review was not completed, the authors were unaware of any randomized control trial or consensus guideline that supports the use of a particular orientation for wound closure strips.

Plastic surgeons pride themselves on precise, multi-layered closures to optimize cosmetic outcomes. Scarring is an important element of wound healing, and is influenced by the type of wound closure method used. It is unclear whether differences in the orientation, number and degree of overlap of wound closure strips influence scar quality. This survey of Canadian plastic surgeons demonstrates a lack of a consensus on whether and how wound closure strips are applied.

\section{Acknowledgements}

The authors gratefully thank Karyn Wagner, executive director of the Canadian Society of Plastic Surgery for her and the Society's support with this project.

\section{References}

1. Autio L, Olson KK. The four S's of wound management: staples, sutures, Steri-Strips, and sticky stuff. Holist. Nurs. Pract. 2002;16(2):80-8.

2. D'ettorre M, Bracaglia R, Gentileschi S, Tambasco D. A trick in steri-strips application: the zig-zag pattern. Int. Wound J. 2013;12(2):233.

3. Plotner AN, Mailler-Savage E, Adams B, Gloster HM. Layered closure versus buried sutures and adhesive strips for cheek defect repair after cutaneous malignancy excision. J. Am. Acad. Dermatol. 2011;64(6):1115-1118.

4. Pedersen V, Struckmann J, Kjaergård H, Geerdsen J, Hansen P. Late cosmetic results of wound closure, strips versus suture. Netherl. J. Surg. 1987;39(5):149-150.

5. Yang S, OzogD. Comparison of Traditional Superficial Cutaneous Sutures Versus Adhesive Strips in Layered Dermatologic Closures on the Back - A Prospective, Randomized, Split-Scar Study. Dermatologic Surg. 2015;41(11):1257-1263.

6. Webster DI, Davis PW. Closure of Abdominal Wounds by Adhesive Strips: A Clinical Trial. Br. Med. J. 1975;3(5985):696-8.

7. Katz KH, Desciak EB, Maloney ME. The optimal application of surgical adhesive tape strips. Dermatologic Surg. 1999;25(9):686-8.

8. Pushpakumar S, Hanson R, Carroll S. The application of Steri-Strips. Plast. Reconstr. Surg. 2004;113(3):1106-7.

9. $3 \mathrm{M}$ Critical \& Chronic \& Division. 3MTM Steri-StripTM Adhesive Skin Closures Application and Removal Pocket Guide (2013). 